\title{
Usefulness of Optical Coherence Tomography Measures as Biomarkers in Schizophrenia
}

\author{
Roberto Rodriguez-Jimenez ${ }^{1,2,3 *}$, Eugenio Blázquez ${ }^{4}$, Antonio Lobo ${ }^{2,5}$, José Luis Santos ${ }^{2,6}$ \\ 'Department of Psychiatry, Instituto de Investigación Sanitaria Hospital 12 de Octubre (imas 12), Madrid, Spain \\ ${ }^{2}$ CIBERSAM (Biomedical Research Networking Centre in Mental Health), Spain \\ ${ }^{3}$ CogPsy-Group, Universidad Complutense de Madrid (UCM), Madrid, Spain \\ ${ }^{4}$ Department of Oftalmology, Hospital Universitario 12 de Octubre, Madrid, Spain \\ ${ }^{5}$ Department of Psychiatry, Universidad Zaragoza, Instituto de Investigación Sanitaria Aragón \\ ${ }^{6}$ Department of Psychiatry, Hospital Virgen de La Luz, Cuenca, Spain
}

Article Info

\section{Article Notes}

Received: July 13, 2018

Accepted: August 09, 2018

\section{${ }^{*}$ Correspondence}

Dr. Roberto Rodriguez-Jimenez, Instituto de Investigación Sanitaria Hospital 12 de Octubre (imas12), Department of Psychiatry, Avda. de Córdoba, s/n, 28041 - Madrid, Spain; Telephone No: +34 91390 8426; Fax No: +34 91390 8538; E-mail: roberto.rodriguez.jimenez@gmail.com

(c) 2018 Rodriguez-Jimenez R. This article is distributed under the terms of the Creative Commons Attribution 4.0 International License

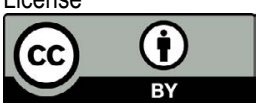

\section{Abstract}

Optical coherence tomography (OCT) is a non-invasive imaging technique recently used to investigate neurodegenerative disorders, such as Alzheimer's disease, Parkinson's disease or multiple sclerosis. Over the last years it has been used in schizophrenia research due to the potential utility of its measurements as biomarkers of the disease. From the first publication by Ascaso et al. (Eur J Psychiat. 2010) until now, a number of papers have been published regarding this topic. Although the majority of them have found a reduction in the thickness or the volume of some retinal and optic nerve structures, there have also been some results with contradictory data. These could be due on the one hand to methodological factors such as the use of different OCT devices or the inclusion/ exclusion criteria. On the other hand, there could be an important limitation due to the fact that the different stages of the disorder and their different neurobiological correlates and biomarkers were not taken into consideration. Future longitudinal studies, that begin the assessments in the first psychotic episode (or even before, in ultra-high risk population) are needed in order to clarify the potential role of some OCT measures as biomarkers in schizophrenia.

\section{Introduction}

Optical coherence tomography (OCT) is a non-invasive imaging technique that provides high-resolution cross-sectional images of the retinal nerve fiber layer (RNFL) and optic nerve head (see figures 1,2). This technique was first described by Huang et al. ${ }^{1}$ from the Massachusetts Institute of Technology. The retina is anatomically and developmentally an extension of the central nervous system (CNS). Due to this fact, the retina is considered to be part of the CNS, and some authors have considered OCT as a "window into the brain"2,3. Several reports have been published showing a significant reduction in RNFL thickness and other retinal structures in some neurological disorders such as Alzheimer's disease ${ }^{4-8}$, mild cognitive impairment ${ }^{7,9-11}$, multiple sclerosis $^{12-14}$, or Parkinson's disease ${ }^{15-20}$. Also, the OCT technique has been used in the study of some neuropsychiatric disorders as depression $^{21,22}$, fibromyalgia ${ }^{23,24}$, anorexia nervosa ${ }^{25}$, bipolar disorder ${ }^{26-28}$, and schizophrenia.

\section{Studies of OCT in Schizophrenia}

Regarding schizophrenia, the first paper published by Ascaso et al. ${ }^{29}$ assessed retinal nerve fiber layer (RNFL) thickness in 10 patients affected by schizophrenia compared with 10 age-matched controls. The authors found that patients with schizophrenia showed a statistically significant 


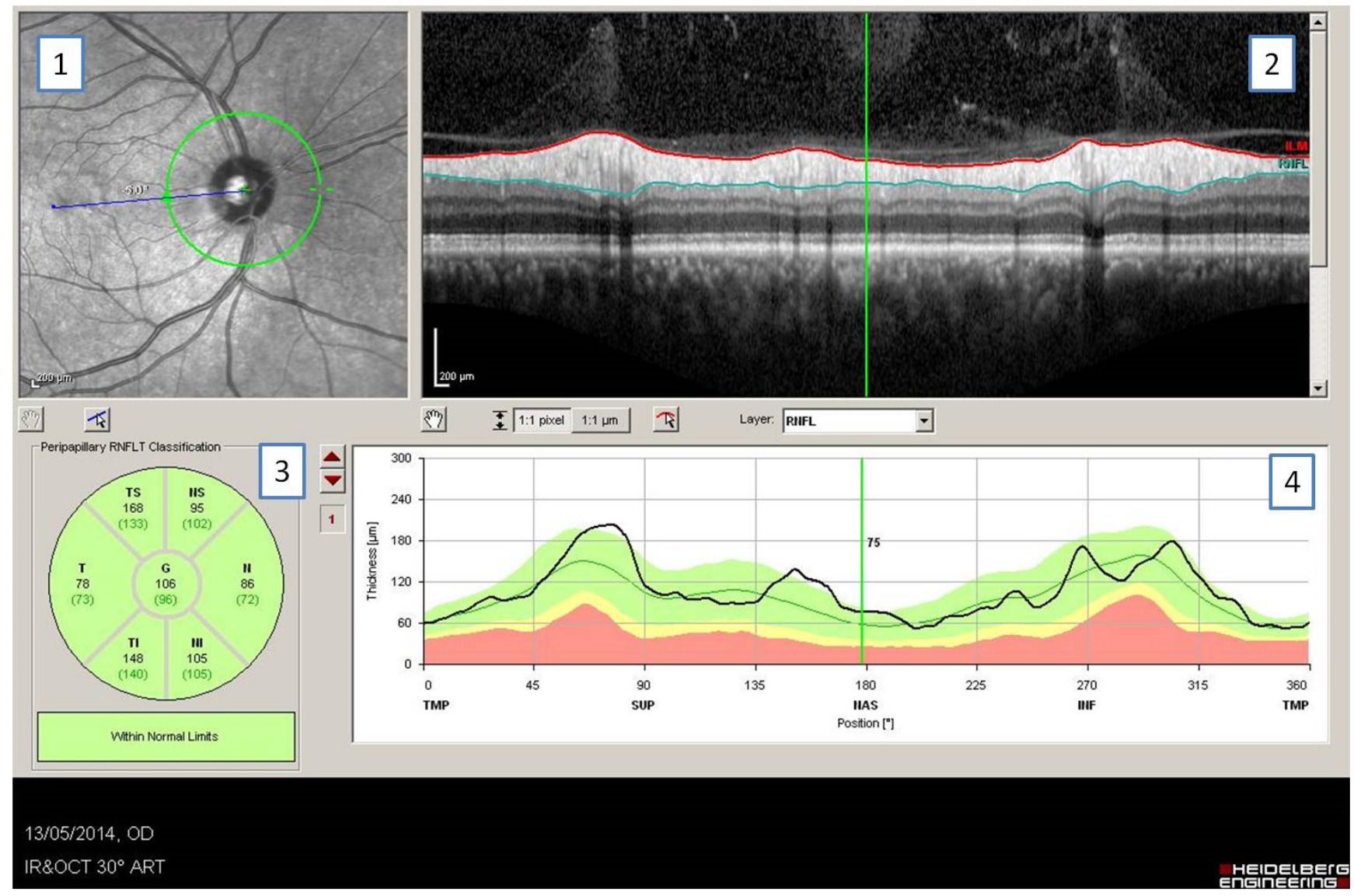

Figure 1: Measurement of retinal nerve fiber layer (RNFL) thicknesses with OCT.

1. A circle is drawn around the optic disc to measure peripapillary RNFL thickness.

2. A picture demonstrating the RNFL.

3. Seven measurements are performed for each eye, providing the RNFL thicknesses of the nasal superior (NS), nasal (N), nasal-inferior

(N) temporal inferior (TI), Temporal (T), temporal superior (TS) and global (G) sectors.

4. RNFL thickness map.

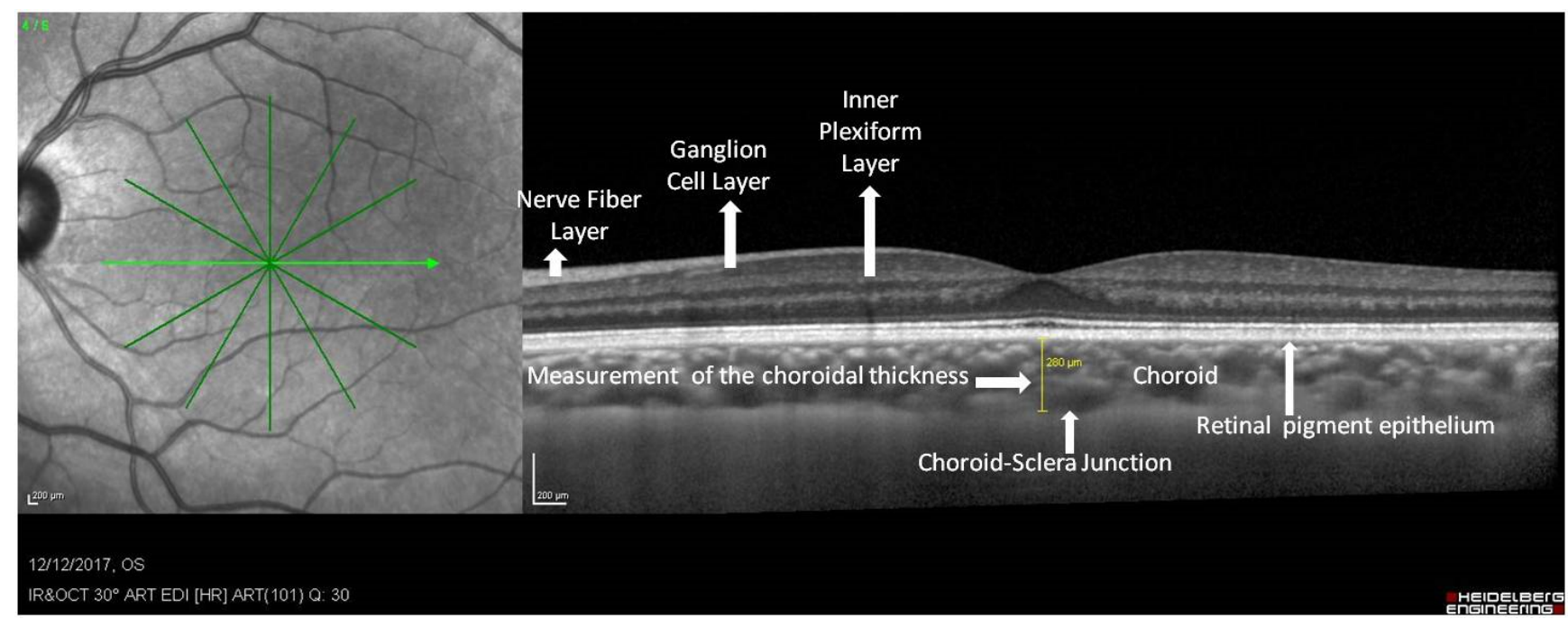

Figure 2: Anatomical correspondence between retinal layers and OCT: Retinal nerve fiver Layer (RNFL), ganglion cell layer (GCL), inner plexiform layer (IPL), retinal pigment epithelium (RPE), Choroid (Ch) and choroid-sclera junction. Measurement of the choroidal thickness: perpendicular line (middle yellow line) is drawn subfoveal from the outer edge of retinal pigment epithelium to the choroidsclera junction. 
reduction of the overall RNFL thickness compared with controls. This reduction was observed in peripapillary RNFL thickness in the nasal quadrant in patients with schizophrenia, however the remaining peripapillary RNFL quadrants, the macular thickness and volume did not reveal differences between both groups. Although they used a small sample, the results were in the line of other studies in neurodegenerative disorders. However, the second published paper on this topic by Chu et al. ${ }^{30}$ did not find similar results. This study was carried out in 49 patients (38 with schizophrenia and 11 with schizoaffective disorder) compared with 40 healthy controls matched for age and gender. The authors found no differences between patients and controls in the whole RNFL thickness and in macular volume. The only difference found was that RNFL in the right nasal quadrant of the schizoaffective patients was thinner than in patients with schizophrenia. In the patient's group, the positive symptom severity assessed with the Schedule for the Assessment of Positive Symptoms (SAPS) was associated with a smaller macular volume. The difference found in these results compared to the work of Ascaso et al. ${ }^{29}$ could be attributed to the fact that they included patients with schizoaffective disorder and with a shorter mean illness duration.

In this controversial scenario, the work of Lee et al. ${ }^{31}$ seemed to support the results of reduction in thickness of retinal structures. It was carried out in 30 patients with schizophrenia and 30 age-matched controls. The authors found that the patients with schizophrenia showed a statistically significant reduction in overall peripapillary RNFL thickness, macular thickness, and macular volume compared with controls. They described that these findings occurred in patients with a duration of more than 2 years (but there were only 5 patients with a duration of illness of less than 2 years in this study). Furthermore, they also found that there was a negative correlation between peripapillary RNFL thickness, macular thickness, and macular volume reduction and the duration of schizophrenic illness. However, in the paper it is not clearly explained whether these results were age-corrected. Age may be a confounding factor since a thinning of retinal structures is observed with increasing age $\mathrm{e}^{32,33}$ and this could affect the results. The study of Yilmaz et al..$^{34}$ also seemed to support the results according to the neurodegenerative hypothesis. They compared measurements of peripapillary RNFL thickness, macular thickness, and macular volume in 34 patients with schizophrenia and 30 randomly selected healthy volunteers. They found that average and nasal RNFL thicknesses were significantly lower in the patient group than in the control group; and macular thickness in the nasal outer and inferior outer quadrants in the patient group were significantly lower than in the control group.

The new work published by Ascaso et al. ${ }^{35}$ introduced a very important methodological improvement: it took into account the clinical stage of 30 patients with schizophrenia. They evaluated the differences between 10 recent illness episode (RIE) schizophrenia patients, 20 non-recent illness episode (NRIE), and 30 matched controls. As a whole group, the patients had a significantly thinner retinal nerve fiber layer (RNFL) in overall measurements, and in the nasal, superior and inferior quadrants. Macular inner ring thickness and macular volume were also significantly lower in patients than in controls. However, when patients were compared with controls, only NRIE (but not RIE) patients had significantly reduced RNFL overall measures, superior RNFL, nasal RNFL, macular volume, and macular inner ring thickness. Thus, retinal parameters observed using OCT in schizophrenia patients could be different according to clinical status. These results, taking into account the clinical stage, could explain the contradictory results found previously. The authors proposed the hypothesis that the ongoing inflammatory processes during the acute psychotic episodes could also affect the retina, increasing its thickness, and that this fact could mask the RNFL thinning in these patients. Finally, contrary to the results of Lee et al. ${ }^{31}$, the authors did not find significant correlation between illness duration and retinal measurements, but in this work the results were controlled for age.

The technological advances in OCT devices resulted in the possibility of segmentation of the retina in the ganglion cell layer (GCL) and the inner plexiform layer (IPL). The GCL is the part of the retina constituted by ganglion cell bodies, and IPL is constituted by ganglion cell dendrites. Celik et al. ${ }^{36}$ carried out a study with RNFL thickness and with the segmentation of the retina in the GCL and the IPL. The authors compared the retinal measurements in 40 treatment-refractory patients with schizophrenia, 41 treatment responsive patients and 41 controls. They also studied the correlation between these measurements and the disease severity (assessed with the Positive and Negative Syndrome Scale (PANSS), the Clinical Global Impression (CGI), the disease duration, and the number of hospitalizations). They found that the global RNFL thickness was decreased in the patients with schizophrenia compared with the controls. Also, this decrement was found in both GCL and IPL volumes. In addition, although no differences were found in the RNFL measurements between the treatment-resistant and treatment-responsive patients, the GCL and IPL volumes were lower in the treatment-refractory patients compared to the treatmentresponsive patients. Finally, regarding the relationship between retinal measurements and clinical variables, they found no correlation between the RNFL and the disease parameters; however significant negative correlations were found between both the GCL and IPL volumes with almost all clinical severity variables. 
A critical point of view regarding the results that found alterations in retinal structures of patients with schizophrenia was shown by Silverstein et $\mathrm{al}^{37}$. They hypothesized that these results could be due to the existence of medical comorbidities frequently present in patients with schizophrenia that affect the retina measurements, such as diabetes or hypertension. In their work, they assessed RNFL, macula and ganglion cell-inner plexiform layer (GCL-IPL) thickness, in 32 patients with schizophrenia and 32 matched controls. They also assessed cup volume and the cup-to-disc ratio at the optic nerve head. The first could be an indicator of the overall size of the optic nerve, and the cup-to-disc ratio, if it is enlarged, can reflect neural tissue loss, vascular abnormalities, inflammation, or an enlarged optic nerve. No differences were found in RNFL, macula, or GCL-IPL thickness. However, thinning of these layers was related to the presence of diabetes or hypertension across the sample as a whole (regardless of whether they had or did not have schizophrenia). Regarding the optic nerve head measurements, the schizophrenia group demonstrated enlarged cup volume and an enlarged cupto-disc ratio in both eyes, which were unrelated to medical comorbidity, but were related to increased cognitive symptoms (assessed using the PANSS cognitive factor). The authors concluded that previous reports of retinal thinning may be artifacts of medical comorbidities and proposed the use of optic nerve head abnormalities as biomarkers of central nervous system abnormalities illnesses, including cognitive dysfunction.

The recent work of Topcu-Yilmaz et al. ${ }^{38}$ (2018) was carried out with inpatients. The authors assessed RNFL, macular, and subfoveal choroidal thickness (CT) in 59 inpatients with schizophrenia and 36 age and gendermatched controls. They found that the macular thickness in the superior inner, nasal inner, inferior inner, temporal inner and temporal outer segments was significantly decreased in schizophrenic patients. However, no differences in RNFL thickness and CT thickness measurements were found between patients and controls. There was no significant correlation found between clinical variables (illness duration, PANSS score, CGI score) and either RNFL thickness or macular thickness measurements. The only correlation, weak and negative, was found between disease duration and CT. The clinical stage of this sample of patients (inpatients) makes it difficult to compare these results with those of studies carried out with outpatients, presumably in the non-acute stage.

More recent advances in OCT scan resolution have allowed the measurement of thickness of all individual retinal layers using image segmentation. Samani et al. ${ }^{39}$ (2018) assessed, for the first time, all individual retinal layers thickness at 3 macular regions in 35 patients with schizophrenia and 50 demographically matched controls. They looked for correlations between retinal layer measurements and symptoms severity, visual contrast sensitivity, illness duration, and the dose of antipsychotic drugs. They found that total retinal and photoreceptor complex thickness was reduced in patients in all regions. Segmentation revealed consistent thinning of the outer nuclear layer and inner segment layer, as well as a pattern of parafoveal ganglion cell changes. The authors found that retinal layer abnormalities were related to negative symptoms and to visual contrast sensitivity. Thus, negative symptom severity was inversely correlated with foveal photoreceptor complex thickness and outer nuclear layer thickness; and low spatial frequency contrast sensitivity was reduced in patients and correlated with temporal parafoveal ganglion cell complex thinning.

\section{Conclusion}

OCT is a non-invasive imaging technique useful for approaching the study of the CNS in some neurodegenerative disorders like Parkinson's disease ${ }^{15-20}$, Alzheimer's disease $\mathrm{e}^{4-8}$, or multiple sclerosis ${ }^{12-14}$. The measurements of the retinal layers, the macular thickness, or the optic nerve head have been proposed as biological markers of neurodegenerative diseases or their evolution ${ }^{40,41}$. OCT applied to schizophrenia has produced interesting results, but some of the published works showed apparently opposite results. In general terms, the results coincide with those found in other neurodegenerative disorders: a reduced thickness in global RNFL (or some of its quadrants) or other retinal layers, and similar reductions in macular thickness and volume and optic nerve abnormalities. However, not all published studies have found similar results.

This could be due to methodological factors such as the use of different OCT devices, differences in the inclusion/ exclusion criteria, or whether they took into account other factors that could affect retinal parameters such as medical or psychiatric comorbidities (diabetes, hypertension, depression) or the antipsychotic treatment. Nonetheless, OCT measures could be useful biomarkers in schizophrenia thanks to their correlation with clinical variables that has been found in some studies, as well as its relatively easy and affordable practicability (for example compared with magnetic resonance imaging). Future studies should take into account the fact that schizophrenia is diagnosed based on a sum of different clinical criteria, and this could lead to different types of schizophrenia with different biomarkers. Moreover, in schizophrenia different clinical stages have been described ${ }^{42,43}$ that could be based in different neurobiological alterations, and this could affect the retinal parameters. Finally, regarding this disease, some studies suggest that it is a neurodevelopment illness ${ }^{44,45}$ while others support a neurodegenerative component (Gupta et al., 2009). Thus, neurodevelopment and neurodegenerative components should be included in the etiopathogenesis of the disorder ${ }^{46}$. Future longitudinal studies, that begin 
assessments in the first psychotic episode (or even before, in ultra-highrisk population) will help us answer some etiopathological questions and clarify the role of these OCT measurements as a biomarkers in schizophrenia.

\section{Acknowledgement}

We are grateful to Dr. Almudena de Pablo for the review of the manuscript.

\section{Declaration of interest}

Dr. R. Rodriguez-Jimenez has been a consultant for, spoken in activities of, or received grants from: Instituto de Salud Carlos III, Fondo de Investigación Sanitaria (FIS), Centro de Investigación Biomédica en Red de Salud Mental (CIBERSAM), Madrid Regional Government (S2010/ BMD2422 AGES), Janssen-Cilag, Lundbeck, Otsuka, Pfizer, Ferrer, Juste, Takeda. The other authors declare that the research was conducted in the absence of any commercial or financial relationships that could be construed as a potential conflict of interest.

\section{Funding/support}

This work was partially supported by Instituto de Investigación Biomédica Hospital 12 de Octubre (imas12).

\section{References}

1. Huang D, Swanson EZ, Lin CP, et al. Optical coherence tomography. Science. 1991; 254: 1178-1181.

2. London A, Benhar I, Schwartz M. The retina as a window to the brainfrom eye research to CNS disorders. Nat Rev Neurol. 2013; 9: 44-53.

3. Gupta S, Zivadinov R, Ramanathan M, et al. Optical coherence tomography and neurodegeneration: are eyes the windows to the brain? Expert Rev Neurother. 2016; 16: 765-775.

4. Parisi V, Restuccia R, Fattapposta F, et al. Morphological and functional retinal impairment in Alzheimer's disease patients. Clin Neurophysiol. 2001; 112: 1860-1867.

5. Berisha F, Feke GT, Trempe CL, et al. Retinal abnormalities in early Alzheimer's disease. Invest Ophthalmol Vis Sci. 2007; 48: 2285-2289.

6. Moreno-Ramos T, Benito-León J, Villarejo A, et al. Retinal nerve fiber layer thinning in dementia associated with Parkinson's disease, dementia with Lewy bodies, and Alzheimer's disease. J Alzheimers Dis. 2013; 34: 659-664.

7. Ascaso FJ, Cruz N, Modrego PJ, et al. Retinal alterations in mild cognitive impairment and Alzheimer's disease: an optical coherence tomography study. J Neurol. 2014; 261: 1522-1530.

8. Ferrari L, Huang SC, Magnani G, et al. Optical coherence tomography reveals retinal neuroaxonal thinning in frontotemporal dementia as in Alzheimer's disease. J Alzheimers Dis.2017; 56: 1101-1107.

9. Shen Y, Liu L, Cheng Y, et al. Retinal nerve fiber layer thickness is associated with episodic memory deficit in mild cognitive impairment patients. Curr Alzheimer Res. 2014; 11: 259-266.

10. Shi Z, Zhu Y, Wang M, et al. The utilization of retinal nerve fiber layer thickness to predict cognitive deterioration. J Alzheimers Dis. 2015 ; 49: 399-405.

11. Liu D, Zhang L, Li Z, Zhang X, et al. Thinner changes of the retinal nerve fiber layer in patients with mild cognitive impairment and Alzheimer's disease. BMC Neurol. 2015; 15: 14
12. Thrower BW. Clinically isolated syndromes: predicting and delaying multiple sclerosis. Neurology. 2007; 68(Suppl 4): S12-15.

13. Sergott RC, Frohman E, Glanzman R, et al. OCT in MS Expert Panel. The role of optical coherence tomography in multiple sclerosis: expert panel consensus. J Neurol Sci. 2007; 263: 3-14.

14. Toledo J, Sepulcre J, Salinas-Alaman A, et al. Retinal nerve fiber layer atrophy is associated with physical and cognitive disability in multiple sclerosis. Mult Scler. 2008; 14: 906-912.

15. Inzelberg R, Ramirez JA, Nisipeanu P, et al. Retinal nerve fiber layer thinning in Parkinson disease. Vision Res. 2004; 44: 2793-2797.

16. Hajee ME, March WF, Lazzaro DR, et al. Inner retinal layer thinning in Parkinson disease. Arch Ophthalmol. 2009; 127: 737-741.

17. Kirbas S, Turkyilmaz K, Tufekci A, et al. Retinal nerve fiber layer thickness in Parkinson disease. J Neuroophthalmol. 2013; 33: 62-65.

18. Jiménez B, Ascaso FJ, Cristóbal JA, et al. Development of a prediction formula of Parkinson disease severity by optical coherence tomography. Mov Disord. 2014; 29: 68-74

19. Satue M, Seral M, Otin S, et al. Retinal thinning and correlation with functional disability in patients with Parkinson's disease. Br J Ophthalmol.2014; 98: 350-355.

20. Garcia-Martin E, Rodriguez-Mena D, Satue M, et al. Electrophysiology and optical coherence tomography to evaluate Parkinson disease severity. Invest Ophthalmol Vis Sci. 2014; 55: 696-705.

21. Kalenderoglu A, Çelik M, Sevgi-Karadag A, et al. Optic coherence tomography shows inflammation and degeneration in major depressive disorder patients correlated with disease severity. J Affect Disord. 2016; 204: 159-65.

22. Ylldız M, Alim S, Batmaz S, et al. Duration of the depressive episode is correlated with ganglion cell inner plexifrom layer and nasal retinal fiber layer thicknesses: optical coherence tomography findings in major depression. Psychiatry Res. 2016; 251: 60-66.

23. Garcia-Martin E, Garcia-Campayo J, Puebla-Guedea $M$, et al Fibromyalgia Is Correlated with Retinal Nerve Fiber Layer Thinning. PLoS One. 2016 Sep 1; 11: e0161574.

24. Ulusoy MO, Kal A, Işik-Ulusoy S, et al. Choroidal thickness in patients with fibromyalgia and correlation with disease severity. Indian J Ophthalmol. 2018; 66: 428-432.

25. Moschos MM, Moustafa GA, Gonidakis F, et al.Retinal and choroidal alterations in patients with anorexia nervosa without vision loss. Int J Eat Disord. 2016; 49: 386-390.

26. Kalenderoglu A, Sevgi-Karadag A, Celik M, et al. Can the retinal ganglion cell layer (GCL) volume be a new marker to detect neurodegeneration in bipolar disorder. Compr Psychiatry. 2016; 67: 66-72.

27. Mehraban A, Samimi SM, Entezari M, et al. Peripapillary retinal nerve fiber layer thickness in bipolar disorder. Graefes Arch Clin Exp Ophthalmol. 2016; 254: 365-371.

28. Khalil MA, Saleh AA, Gohar SM, et al. Optical coherence tomography findings in patients with bipolar disorder. J Affect Disord. 2017; 218: 115-122.

29. Ascaso FJ, Cabezon L, Quintanilla MA, et al. Retinal nerve fiber layer thickness measured by optical coherence tomography in patients with schizophrenia: A short report. Eur J Psychiat. 2010; 24: 227-235.

30. Chu EM, Kolappan M, Barnes TR, et al. A window into the brain: an in vivo study of the retina in schizophrenia using optical coherence tomography. Psychiatry Res. 2012; 203: 89-94.

31. Lee WW, Tajunisah I, Sharmilla $\mathrm{K}$, et al. Retinal nerve fiber layer structure abnormalities in schizophrenia and its relationship to disease state: evidence from optical coherence tomography. Invest Ophthalmol Vis Sci. 2013; 54: 7785-7792. 
32. Alamouti B, Funk J. Retinal thickness decreases with age: an OCT study. Br J Ophthalmol. 2003; 87: 899-901.

33. Parikh RS, Parikh SR, Sekhar GC, et al. Normal age-related decay of retinal nerve fiber layer thickness. Ophthalmology. 2007; 114: 921926.

34. Yllmaz U, Küçük E, Ülgen A, et al. Retinal nerve fiber layer and macular thickness measurement in patients with schizophrenia. Eur J Ophthalmol. 2016; 26: 375-378

35. Ascaso FJ, Rodriguez-Jimenez R, Cabezón L, et al. Retinal nerve fiber layer and macular thickness in patients with schizophrenia: Influence of recent illness episodes. Psychiatry Res. 2015; 229: 230-236.

36. Celik M, Kalenderoglu A, Sevgi Karadag A, et al. Decreases in ganglion cell layer and inner plexiform layer volumes correlate better with disease severity in schizophrenia patients than retinal nerve fiber layer thickness: Findings from spectral optic coherence tomography. Eur Psychiatry. 2016; 32: 9-15.

37. Silverstein SM, Paterno D, Cherneski L, et al. Optical coherence tomography indices of structural retinal pathology in schizophrenia. Psychol Med. 2017; 1-11.

38. Topcu-Yilmaz P, Aydin M, Ilhan BC. Evaluation of retinal nerve fiber layer, macular, and choroidal thickness in schizophrenia: spectral optic coherence tomography findings. Psychiatry Clin Psycho. 2018; 1-6.

39. Samani NN, Proudlock FA, Siram V, et al. Retinal layer abnormalities as biomarkers of schizophrenia. Schizophr Bull. 2018; 44: 876-885
40. Mirzaei M, Gupta VB, Gupta VK. Retinal changes in Alzheimer's disease: Disease Mechanisms to Evaluation perspectives. J Neurol Neuromed. 2018; 3: 11-13.

41. Lad EM, Mukherjee D, Stinnett SS, et al. Evaluation of inner retina layers as biomarkers in mild cognitive impairment to moderate Alzheimer's disease. PLoS One. 2018; 13: e0192646.

42. Wood SJ, Yung AR, McGorry PD, et al. Neuroimaging and treatment evidence for clinical staging in psychotic disorders: from the at-risk mental state to chronic schizophrenia. Biol Psychiatry. 2011; 70: 619625.

43. Berendsen S, van der Paardt J, van Bruggen M, et al. Exploring construct validity of clinical staging in schizophrenia spectrum disorders in an acute psychiatric ward. Clin Schizophr Relat Psychoses. 2018. [Epub ahead ofprint].

44. Brans RG, van Haren NE, van Baal GC, et al. Longitudinal MRI study in schizophrenia patients and their healthy siblings. Br J Psychiatry. 2008; 193: 422-423.

45. Gupta CN, Calhoun VD, Rachakonda S, et al. Patterns of gray matter abnormalities in schizophrenia based on an international megaanalysis. Schizophr Bull. 2015; 41: 1133-1142.

46. Gupta S, Kulhara P. What is schizophrenia: A neurodevelopmental or neurodegenerative disorder or a combination of both? A critical analysis. Indian J Psychiatry. 2009; 51: 21-27. 